\title{
Amorphous Carbon Assisted Highly Percentage Synthesis of Ultralong, Well-Aligned, and Visible Carbon Nanotubes
}

\author{
N. K. Chang ${ }^{1}$, C. C. Chuang ${ }^{1}$, C. C. Su$^{1}$, S. H. Chang ${ }^{1,2}$ \\ ${ }^{1}$ Department of Mechanical Engineering, National Taiwan University, Taipei 106, Taiwan, ROC \\ ${ }^{2}$ MIRL, Industrial Technology Research Institute, Hsin Chu 310, Taiwan, ROC. \\ No. 1, Sec. 4, Roosevelt Rd., Taipei, Taiwan, TEL:886-3-5919388, FAX:886-3-5820235 \\ shuochang@, itri.org.tw
}

\section{Abstract}

In this study, we present a novel method to observe the location and the length of carbon nanotubes under an optical system instead of SEM. Firstly, the carbon nanotubes were synthesized by using a mixed methane and ethylene carbon source in the chemical vapor deposition (CVD) [1]. Then, the carbon nanotubes were coated with amorphous carbon by blowing ethanol vapor into the furnance at $900{ }^{\circ} \mathrm{C}$. The observations of SEM and TEM show that each carbon fiber consists of one carbon nanotube and a great deal of amorphous carbon. The diameters of the carbon fibers, around $500 \mathrm{~nm}$, are so large that they can be easily observed and estimated from the optical images, as shown in Figure 2. For example, the longest length of carbon fiber observed in this experiment can exceed $2 \mathrm{~cm}$. In addition, the most of the carbon fibers are well-aligned due to floating catalyst [2]. This method will be useful as locating of suspended carbon nanotubes or manipulating nanotubes under the optical system.
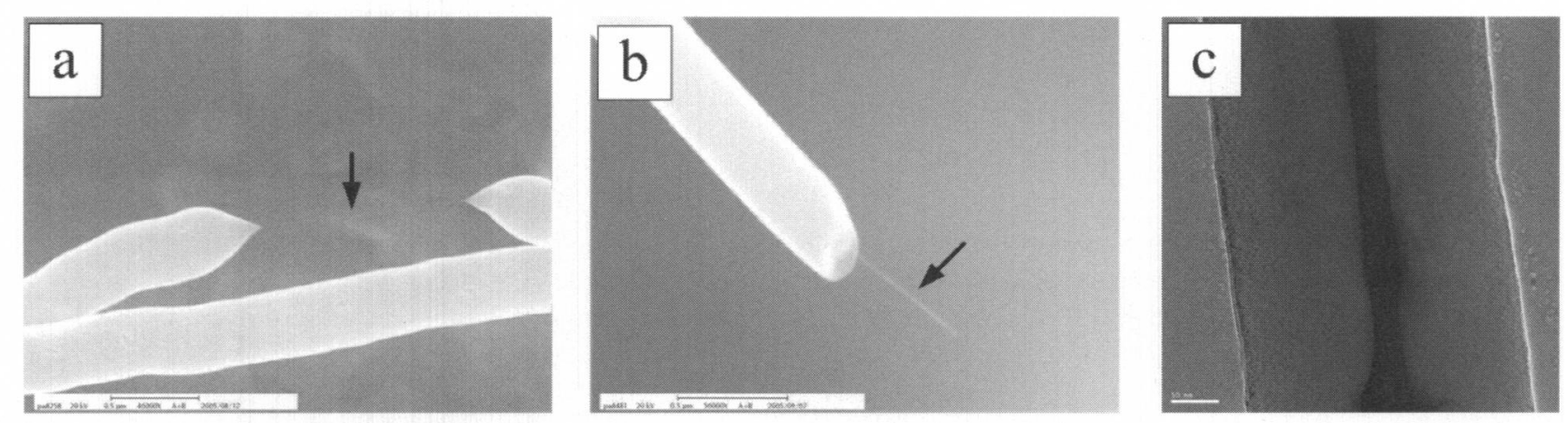

Figure 1. The SEM and TEM images of carbon nanotubes with a considerable amount of amorphous carbon. (a) One of the fibers is a carbon nanotube coated with part of amorphous carbon, and another one is a carbon nanotube completely coated with amorphous carbon. (b) The carbon fiber was purposely broken via AFM tip, and then the nanotube was pulled out. (c) The cross-sectional TEM image of the carbon fiber.
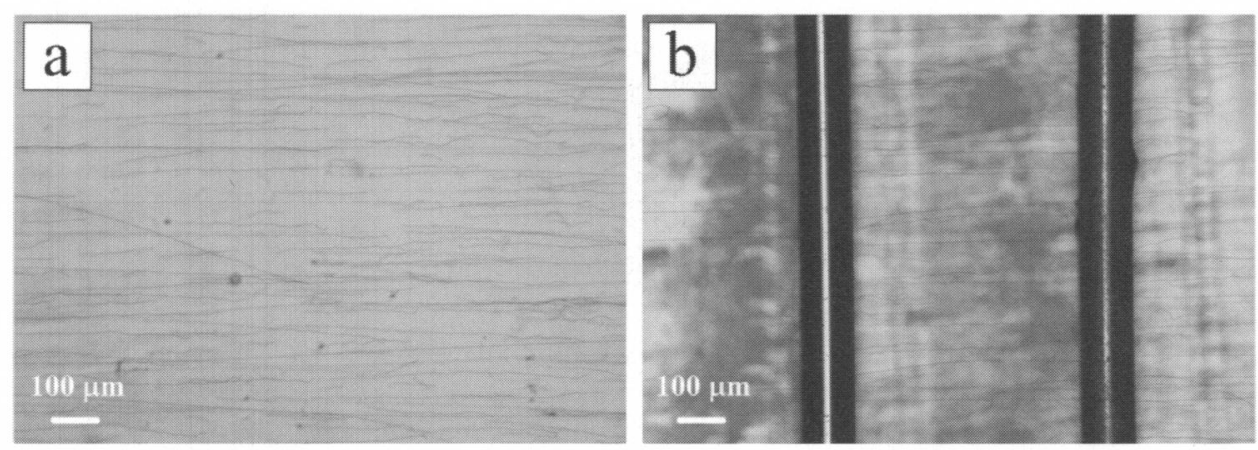

Figure 2. The optical images of well-aligned carbon fibers. (a) The well-aligned carbon fibers lay on 
wafer. The gas flow direction was from right to left. (b) The fiber can suspend between the two quartz fibers which were put on wafer before process of growing the nanotubes.

\section{Reference}

[1] Woong Kim, Hee Cheul Choi, Moonsub Shim, Yiming Li, Dunwei Wang, and Hongjie Dai, “ Synthesis of Ultralong and High Percentage of Semiconducting SWCNT," Nano Letters, Vol. 2, No. 7, pp. 703-708, 2002.

[2] Shaoming Huang, Xianyu Cai, Chunsheng Du, and Jie Liu, "Oriented Long Single Walled Carbon Nanotubes on Substrates From Floating Catalysts," The Journal of Physical Chemistry B, Vol. 107, No. 48, pp. 13251-13254, 2003. 